\title{
Synergistic inhibition of colon cancer cell growth with nanoemulsion-loaded paclitaxel and PI3K/ mTOR dual inhibitor BEZ235 through apoptosis
}

This article was published in the following Dove Press journal:

International Journal of Nanomedicine

5 May 2016

Number of times this article has been viewed

\author{
Hong Zou ${ }^{1,2}$ \\ Li Li' \\ Ines Garcia Carcedo' \\ Zhi Ping $X u^{\prime}$ \\ Michael Monteiro' \\ Wenyi $\mathrm{Gu}^{\prime}$
}

'Australian Institute for Bioengineering and Nanotechnology, The University of Queensland, St Lucia, Brisbane, QLD, Australia; ${ }^{2}$ Department of Pathology, Key Laboratory for Xinjiang Endemic and Ethnic Diseases, Shihezi University School of Medicine, Shihezhi University, Xinjiang, People's Republic of China
Correspondence: Wenyi Gu Australian Institute for Bioengineering and Nanotechnology, The University of Queensland, Corner of Cooper Road, St Lucia, Brisbane, QLD 4072, Australia Tel +61733464168

Fax +6I 733463973

Email w.gu@uq.edu.au
Abstract: Colon cancer is the third most common cancer in the world, with drug resistance and metastasis being the major challenges to effective treatments. To overcome this, combination therapy with different chemotherapeutics is a common practice. In this study, we demonstrated that paclitaxel (PTX) together with BEZ235 exhibited a synergetic inhibition effect on colon cancer cell growth. Furthermore, nanoemulsion (NE)-loaded PTX and BEZ235 were more effective than the free drug, and a combination treatment of both NE drugs increased the efficiency of the treatments. BEZ235 pretreatment before adding PTX sensitized the cancer cells further, suggesting a synergistic inhibition effect through the phosphatidylinositol-3-kinases/protein kinase B/mammalian target of rapamycin pathway. The 50\% inhibitory concentrations for BEZ235 were $127.1 \mathrm{nM}$ and $145.0 \mathrm{nM}$ and for PTX $9.7 \mathrm{nM}$ and $9.5 \mathrm{nM}$ for HCT-116 and HT-29 cells, respectively. When loaded with NE the $50 \%$ inhibitory concentrations for BEZ235 decreased to $52.6 \mathrm{nM}$ and $55.6 \mathrm{nM}$ and for PTX to $1.9 \mathrm{nM}$ and $2.3 \mathrm{nM}$ for HCT-116 and HT-29 cells, respectively. Combination treatment with $10 \mathrm{nM}$ NE-BEZ235 and $0.6 \mathrm{nM}$ and $1.78 \mathrm{nM}$ NE-PTX could kill 50\% of HCT116 and HT-29, respectively. The cell death caused by the treatment was through apoptotic cell death, which coincided with decreased expression of anti-apoptotic protein B-cell lymphoma 2. Our data indicate that the combination therapy of PTX with the phosphatidylinositol-3-kinases/ protein kinase B/mammalian target of rapamycin dual inhibitor BEZ235 using NE delivery may hold promise for a more effective approach for colon cancer treatment.

Keywords: combination therapy, signal pathway inhibitor, chemotherapy, nanomedicine

\section{Introduction}

Colon cancer is the third most common cancer in humans and has a high mortality rate. Like many other cancers, drug resistance and metastasis are the biggest hurdles for effective treatments. For metastasized cases, the 5 -year survival rate is only $\sim 10 \% .{ }^{1}$ To overcome this, a common clinical practice is to use combination therapy, particularly the combination of two or three different chemotherapeutics, though other approaches including radiotherapy, targeted therapy, and immune therapy can also be used. It is found that most of these approaches, especially chemotherapy, have severe side effects. The development of advanced nanodrug delivery systems can thus offer a solution by increasing the drug's efficiency (including increased solubility), thereby reducing the drug dosage.

Paclitaxel (PTX) is a common chemotherapeutic that has been used clinically for many years to treat ovarian, breast, lung, and pancreatic cancer. It is also an approved chemotherapeutic in the UK for ovarian, breast, lung, bladder, prostate, esophageal, and other types of solid tumors as well as melanoma and Kaposi's sarcoma. ${ }^{2}$ The mechanism of action of PTX involves interference with the process of normal 
breakdown of microtubules during cell division, causing apoptosis in the treated cells. ${ }^{3-5}$ But the low solubility of PTX hinders its application in the clinic. In addition, PTX can cause some side effects such as hypersensitivity reactions, myelosuppression, and peripheral neuropathy. ${ }^{6}$ This makes the nanodrug delivery system a useful tool to assist with PTX solubility and delivery.

There have been many studies on the nanodelivery of PTX; for example, albumin-modified PTX (nab-PTX) has been shown to have an improved efficiency compared to the unmodified free drug. ${ }^{6-8}$ Lipid nanocapsule-loaded PTX was also shown to be more effective for oral administration with enhanced bioavailability. ${ }^{9}$ Nanoemulsion (NE) is another attractive way to deliver the hydrophobic drugs such as PTX, and some studies have demonstrated that NE-loaded PTX (NE-PTX) can overcome drug resistance, ${ }^{10,11}$ increase cellular uptake, ${ }^{12,13}$ and enhance cytotoxicity and apoptosis in different cancer cells. ${ }^{14}$ In addition, other nanodelivery systems such as polymeric micelles, hydrogels, and liposomes were also reported to effectively deliver PTX for cancer therapy. ${ }^{15}$

In colon cancer, several previous studies have shown that PTX could induce remarkable G2/M phase arrest ${ }^{16}$ and the activation of caspase 8 apoptosis pathway ${ }^{17}$ in cancer cells. One study also reported that PTX delivered by polymer nanoparticles was 13 times more effective than the free drug in HT-29 cells. ${ }^{18}$ In addition, PTX was used in combination therapy with other chemotherapeutics such as gemcitabine and mitogen-activated protein kinase kinase inhibitors in colon cancer treatment. ${ }^{16,19}$ These data suggest that PTX is a promising chemotherapeutic for colon cancer.

The phosphatidylinositol-3-kinases (PI3K)/protein kinase B (Akt)/mammalian target of rapamycin (mTOR) survival pathway is highly upregulated in cancer cells and plays an important role in cancer development, including colon cancer. ${ }^{20-23}$ Targeted therapy against this pathway has been extensively studied in colon cancer. ${ }^{24}$ This pathway is also associated with drug resistance to chemotherapy drugs. ${ }^{24,25}$ BEZ235 is a relatively new inhibitor of the PI3K/Akt/mTOR pathway and is a promising chemotherapy drug due to its effectiveness and low side effects. ${ }^{26-28}$ It inhibits the activity of both PI3K and mTOR and blocks the feedback activation of PI3K activity by simultaneous inhibition of mTOR. A previous study showed that BEZ235 was effective in inhibiting the growth of both PIK3CA-mutated and -nonmutated colon cancer cell lines. ${ }^{29}$ Recently, we showed that in a combinational therapy of BEZ235 and the chemotherapeutic 5-fluorouracil (5-FU), BEZ235 treatment could sensitize the colon cancer cells HCT-116 to 5-FU-induced apoptosis. Nanoparticle layered double hydroxide-delivered 5-FU was even more effective. ${ }^{30}$
Therefore, we believe that BEZ235 will soon be included in the present treatment regime of colon cancer.

Given that PTX and BEZ235 react to different aspects of cell division of cancer cells, we hypothesized that they might have a synergistic effect on inhibiting the cancer cell growth of colon cancer. Indeed, limited information on the combinational therapy of BEZ235 and PTX in ovarian, ${ }^{31}$ gastric, ${ }^{7}$ pancreatic, ${ }^{32}$ and thyroid ${ }^{33}$ cancers suggests a synergistic inhibitory effect on cancer cell growth through an induced apoptosis. However, this combination therapy has not been documented in colon cancer. In addition, combination treatment with both NE-delivered BEZ235 and NE-delivered PTX has not been reported in any cancer. Therefore, in this study, we first investigated the combination treatment effect of PTX and BEZ235 on the colon cancer cell lines HCT-116 and HT-29, followed by a study of NE-loaded drugs. We found that the combination treatment could synergistically increase cancer cell death through both cell-cycle changes and apoptosis pathways. NE-loaded drugs were even more effective at inhibiting the cancer cell growth.

\section{Materials and methods Preparation and characterization of NE-PTX and NE-BEZ235}

PTX- and BEZ235-encapsulated NEs (NE-PTX and NE-BEZ235) were prepared via low-energy spontaneous emulsification. In brief, PTX or BEZ235 was dissolved in dimethyl sulfoxide and then mixed with capryol 90 , Tween 20, and propylene glycol via vortexing. ${ }^{12}$ The emulsifier mixture (the volume ratio of Tween 20 to propylene glycol was 2:1) and water were added to the oil mixture under vortexing for 5 minutes at the highest speed. The mass ratio of capryol 90, Tween 20, and propylene glycol to water in the NE was 20:35:45. The final concentration of PTX and BEZ235 in NEs was $0.5 \mathrm{mM}$ and $1 \mathrm{mM}$, respectively. The mean droplet size and the size distribution of the as-formulated NEs were determined by dynamic light scattering (Nano ZS, Zetasizer, Nanoseries; Malvern Instruments, Malvern, UK).

\section{Cell lines and 3-(4,5-dimethylthiazol-2-yl)- 2,5-diphenyltetrazolium bromide cell viability assay}

The colon cancer cell lines HCT-116 and HT-29 were commercially purchased from American Type Culture Collection during the years 2010-2012 (Category number CCL-247 and HTB-38; Manassas, VA, USA). Supplemented with $10 \%$ fetal calf serum, $1 \%$ penicillin, and $1 \%$ streptomycin in $75 \mathrm{~mL}$ flasks at $37^{\circ} \mathrm{C}$ and $5 \% \mathrm{CO}_{2}$. The Ethics Committee 
of The University of Queensland does not require ethics approval to be sought for the use of human cell lines. For the cytotoxicity assay, cells were trypsinized for 5 minutes and resuspended in the medium to dilute the concentration to $1 \times 10^{5} / \mathrm{mL}$. Then $100 \mu \mathrm{L}$ of the cells was added to each well of a 96-well plate for the cytotoxicity assay under treatments of various concentrations of BEZ235 and PTX. After a 48-hour treatment, 3-(4,5-dimethylthiazol-2-yl)2,5-diphenyltetrazolium bromide (MTT) assay was used to measure cell viability as per the manufacturer's instructions (Sigma-Aldrich Co., St Louis, MO, USA).

\section{Cell cycle analysis and $\beta$-tubulin staining}

Propidium iodide (PI) was used to stain the DNA content. HCT-116 and HT-29 cells were harvested by trypsinization and were washed with $1 \times$ phosphate-buffered saline (PBS). The cells were resuspended in PBS as $2 \times 10^{6} / \mathrm{mL}$. In $1 \mathrm{~mL}$ of such cell suspension, $1 \mathrm{~mL}$ of $100 \%$ cold ethanol was added dropwise and vortexed gently. The mixture was stored at $-20^{\circ} \mathrm{C}$ overnight. After washing the cells with cold PBS once, they were resuspended in $300 \mu \mathrm{L} \mathrm{PI/Triton} \mathrm{buffer} \mathrm{containing}$ 0.01\% (v/v) Triton 100 (Sigma-Aldrich Co.) and $6 \mu \mathrm{g}$ PI. After incubation at room temperature for 40 minutes, the stained cells were analyzed using the flow cytometry (Accuri; BD, Franklin Lakes, NJ, USA) with FlowJo software.

For $\beta$-tubulin staining, the cells were set up in a sixwell plate at $2 \times 10^{5}$ cells/well a day before treatment. The drugs were directly added to the medium the next day, and after a 2-hour treatment, the medium was removed and the cells were washed with $1 \times$ PBS followed by fixation with $4 \%$ paraformaldehyde/PBS. The cells were stained with 1:200 diluted mouse antihuman $\beta$-tubulin antibody (Cell Signaling Technology, Danvers, MA, USA) followed by 1:500 diluted secondary goat antimouse immunoglobulin G (IgG) (heavy chain and light chain) conjugated with Texes Red $^{\text {TM }}$ (Sigma-Aldrich Co.). The cell nuclei were stained with 4',6-diamidino-2-phenylindole and viewed under a fluorescent microscope.

\section{Cell apoptosis analysis}

Cell apoptosis and cell death were detected using an fluorescein isothiocyanate-Annexin V apoptosis Detection Kit I from $\mathrm{BD}$ (Pharmingem ${ }^{\mathrm{TM}}$ ) following the procedure recommended by the manufacturer and as previously reported..$^{30}$ HCT-116 and HT-29 cells were cultured in six-well plates with $2 \times 10^{5}$ cells in each well. Cells were treated for 24 hours with the concentrations of BEZ235 at $50 \mathrm{nM}$ and of PTX from $2 \mathrm{nM}$ to $4 \mathrm{nM}$. Combination treatments of BEZ235 and PTX were also carried out. The treated cells were collected by centrifugation, suspended in $100 \mu \mathrm{L}$ of the provided buffer, and stained with fluorescein isothiocyanate-Annexin $\mathrm{V}$ conjugates and PI for 15 minutes. Cell death and apoptosis were then detected by the flow cytometry (Accuri; BD) and analyzed using the FlowJo software.

\section{Western blotting}

Cells from cultures were lysed in lysis and extraction buffer (Cell Signaling Technology) containing $2 \mu \mathrm{L} / \mathrm{mL}$ protease inhibitor cocktail (Sigma-Aldrich Co.). Proteins were separated by electrophoresis in 10-well gels by a Bio-Rad apparatus at $120 \mathrm{~V}$ for 1.5 hours. The separated proteins in the gel were transferred onto a poly(vinylidene fluoride) membrane at $100 \mathrm{~V}$ for 1 hour. The membrane was blocked at room temperature with $5 \%$ bovine serum albumin in Tris-buffered saline and 0.5\% Tween 20 (TBST) buffer for 1 hour and washed three times with TBST, with each wash lasting for 5 minutes. The membrane then was incubated overnight with rabbit antihuman B-cell lymphoma 2 (Bcl-2) antibody (Cell Signaling Technology) at 1:500 or 1:2,000 diluted antihuman $\beta$-tubulin antibody (Cell Signaling Technology). After washing three times with TBST, the membrane was incubated for 2 hours at room temperature with horseradish-peroxidase-conjugated goat antirabbit antibody (Cell Signaling Technology) at the dilution of 1:2,500. The membrane was incubated with enhanced chemiluminescence for 5 minutes and scanned.

\section{Data analysis}

Data collected from experimental and control groups were expressed as mean $\pm \mathrm{SD}$. The one-way analysis of variance and the unpaired Student's $t$-test (GraphPad Prism 6 program) were used to analyze the differences between groups and discriminate the significant differences (two-tail, $P<0.05$ ) between experimental and control groups.

\section{Results Characterization of NE-PTX and NE-BEZ235}

The homogenous NE-PTX and NE-BEZ235 were prepared by low-energy spontaneous emulsification. At an oil/emulsifier/ water ratio of 20:35:45, a clear and uniform suspension was obtained. The dynamic light scattering results in Figure 1 show that the NE system had a narrow size distribution with the $Z$-average size of $171.1 \mathrm{~nm}$ and polydispersity index of 0.32. After loading the drugs, the size and polydispersity index of the drug-NE remained unchanged. The final concentrations of NE-PTX and NE-BEZ235 were $0.5 \mathrm{mM}$ and $1.0 \mathrm{mM}$, respectively. 


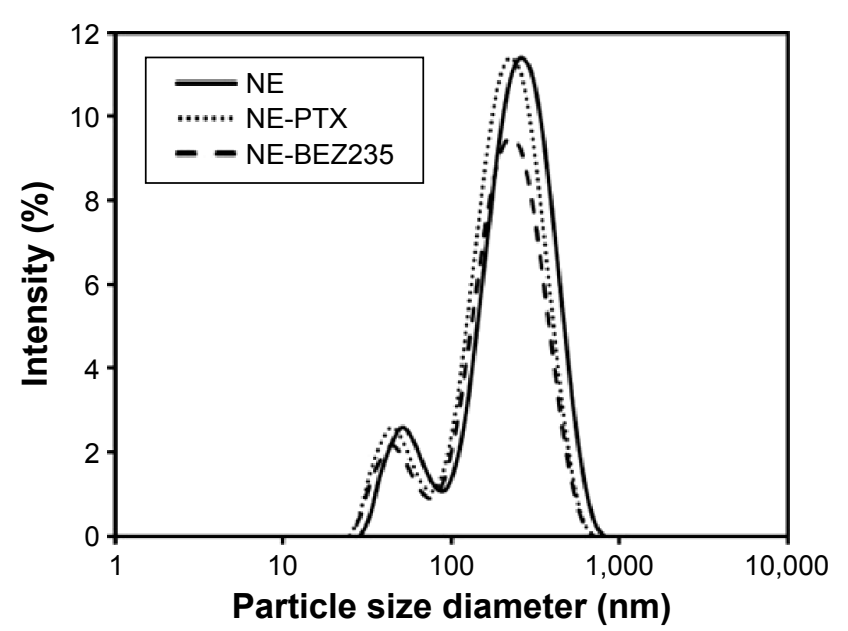

Figure I Particle size of NE: the particle size distributions of NE, NE-PTX, and NE-BEZ235 from DLS analysis.

Abbreviations: DLS, dynamic light scattering; NE, nanoemulsion; PTX, paclitaxel.
Inhibition of colon cancer cell growth by PTX, BEZ235, and combination

\section{treatment}

To determine the combination treatment dose of each drug in each cell line, we first examined the dose-dependent inhibitory effect of each drug. As shown in Figure 2, both PTX and BEZ235 showed a dose-dependent inhibitory effect on the cell growth of colon cancer cell lines HCT-116 and HT-29 (Figure 2A and B). The 50\% inhibitory concentration (IC50) values of BEZ235 on HCT-116 and HT-29 cells were $127.1 \mathrm{nM}$ and $145.1 \mathrm{nM}$, respectively, and that of PTX on HCT-116 was $9.7 \mathrm{nM}$ and on HT-29 was $9.5 \mathrm{nM}$ (Table 1). The two cell lines showed a very similar sensitivity to each drug. However, both cell lines seemed more sensitive to PTX than BEZ235 treatment.
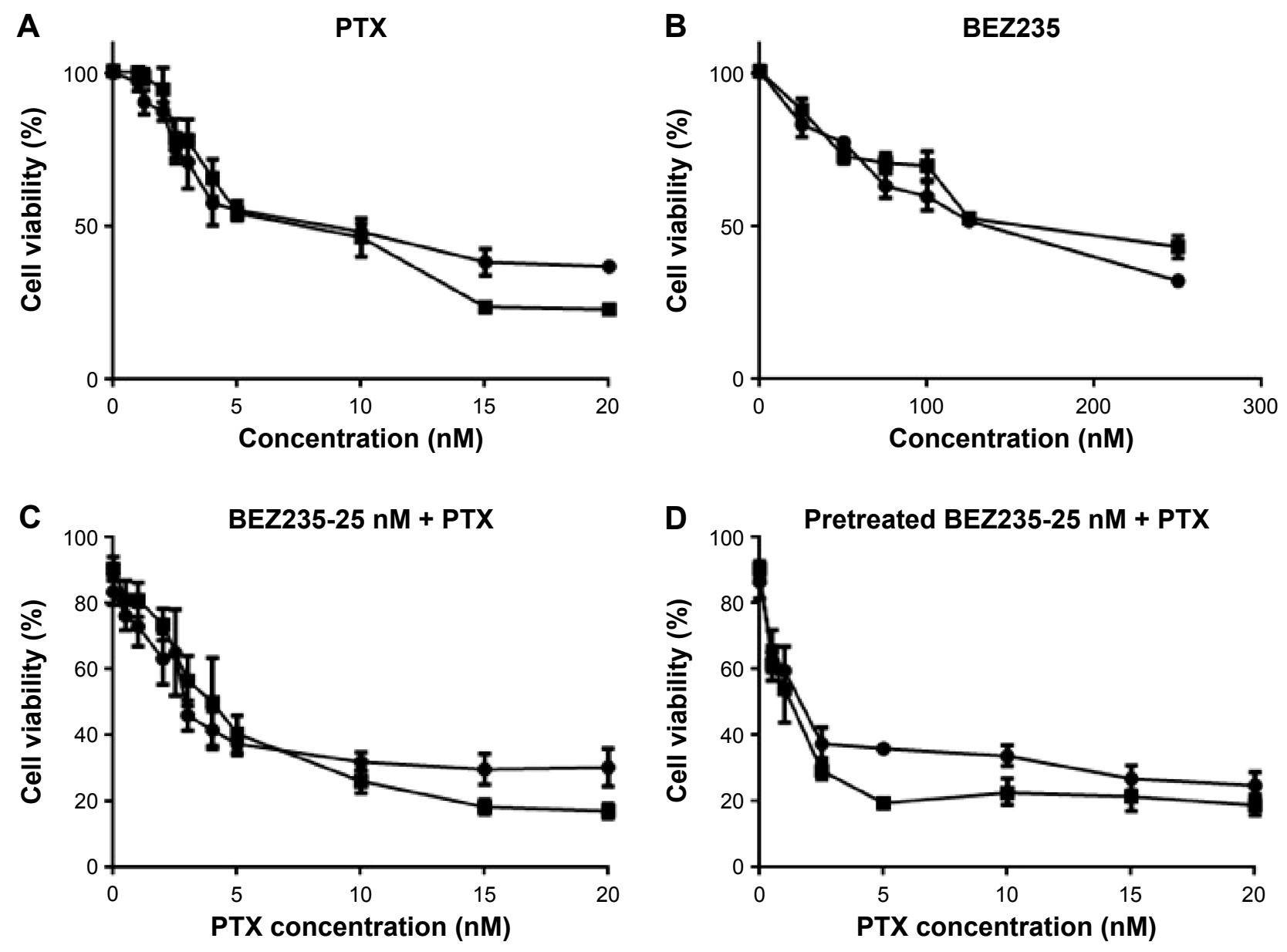

HCT-116

HT-29

Figure 2 Dose-dependent inhibitory effect of PTX and BEZ235 on colon cancer cells.

Notes: (A) MTT assay results on colon cancer cells HCT-II6 and HT-29 treated with PTX at various concentrations (in nM). The two cell lines have a similar sensitivity to the drug treatment. (B) MTT assay results of BEZ235 in the two cell lines at different concentrations (in $\mathrm{nM}$ ). The two cell lines show no differences in sensitivity to the treatment. (C) Combination treatment with a fixed BEZ235 concentration of $25 \mathrm{nM}$ and various PTX concentrations. At $25 \mathrm{nM}$ of BEZ235, both cell lines exhibit an increased sensitivity to PTX treatment. (D) Further increased cell sensitivity to PTX by pretreatment of the cells with BEZ235 for 2 hours followed by adding the same amount of PTX. Abbreviations: MTT, 3-(4,5-dimethylthiazol-2-yl)-2,5-diphenyltetrazolium bromide; PTX, paclitaxel. 
Table I IC50s of free or NE-loaded BEZ235 and PTX in colon cancer treatment

\begin{tabular}{lll}
\hline Treatment & HCT-I I6 (nM) & HT-29 (nM) \\
\hline PTX & 9.72 & $9.5 \mathrm{I}$ \\
BEZ235 & 127.13 & 145.09 \\
BEZ235-25 nM + PTX & 3.22 & 3.92 \\
NE-PTX & 1.91 & 2.23 \\
NE-BEZ2235 & 52.18 & 56.60 \\
NE-BEZ235-I0 nM + NE-PTX & 0.64 & 1.78 \\
NE-BEZ235-I0 nM + PTX & 3.24 & 5.26 \\
BEZ235-25 nM + NE-PTX & 1.12 & 1.89 \\
\hline
\end{tabular}

Abbreviations: IC50, 50\% inhibitory concentrations; NE, nanoemulsion; PTX, paclitaxel.

Figure 2C shows that the combination treatment of PTX and BEZ235 produced a synergistic cytotoxic effect on both cell lines. With $25 \mathrm{nM}$ (five times $<\mathrm{IC50}$ ) of BEZ235, the IC50 of PTX reduced to $3.22 \mathrm{nM}$ for HCT-116 and $3.92 \mathrm{nM}$ for HT-29. These IC50s were almost three times lower than both PTX and BEZ235 treatment alone. Therefore, it seems that BEZ235 can increase the sensitivity of both cells to PTX treatment. These data clearly show that a combination treatment with BEZ235 and PTX remarkably suppresses the growth of both cell lines. To further confirm that the PI3K/Akt/ mTOR pathway is important for PTX treatment, we carried out a pretreatment assay in which the cells were treated with $25 \mathrm{nM}$ BEZ235 for 2 hours before adding PTX. We found that the pretreatment further increased the sensitivity of cancer cells to PTX (Figure 2D), suggesting that this pathway is important for the cytotoxicity induced by PTX in colon cancer.

\section{NE-loaded drugs were even more effective at inhibiting cancer cell growth}

Chemotherapy normally causes severe side effects, even in combination treatments with different drugs. Nanodelivery of these drugs may provide a solution to reduce these side effects by further decreasing the dosage. To achieve this, we used NE to load BEZ235 and PTX to further increase their efficacy. The results showed that the NE-loaded drugs had increased inhibitory effect on both cell lines (Figure 3A and B) with a large decrease in the IC50s; for BEZ235, it was reduced two to three times, and for PTX, it was reduced four to five times (Table 1). The combination treatment of $10 \mathrm{nM}$ NE-BEZ235 and PTX could further reduce the IC50 of PTX to $0.6 \mathrm{nM}$ for HCT-116 and to $1.7 \mathrm{nM}$ for HT-29 cells (Table 1, Figure 3C). $\mathrm{NE}$ alone did not show much cytotoxicity, even at $120 \mathrm{nM}$ (Figure 3D). Low dosages such as these could certainly reduce the side effects in the clinic. These data further confirm that there is a synergistic treatment effect on colon cancer.

\section{PTX and BEZ235 treatment induced cell morphology change}

Apart from the cell viability reduction (measured by MTT assay) caused by the drug treatments, under the light microscope, we observed significant cell death in the wells of combination treatment after 24 hours (Figure 4A). The dead cell numbers were always consistent with MTT results. In addition, we observed morphology changes for both cells after treatment. Using $\beta$-tubulin staining, we could see that the round-shaped cells in controls became more fibroblastshaped cells in the treatment samples (Figure 4B).

\section{Combination treatment caused more sub-GI cell death}

It was reported that BEZ235 could induce cell cycle G1 arrest in the prostate cancer cell line PC $3 \mathrm{M} .{ }^{34} \mathrm{PTX}$ was also shown to arrest ovarian cancer cells at the G2/M phase of cell cycle. ${ }^{35}$ To examine whether the combination treatment with PTX and BEZ235 enhanced this effect on the cell cycle of HCT-116 and HT-29, we carried out a cell cycle analysis. The results showed that the combination treatment remarkably increased the sub-G1 (apoptotic) cell population to $40.5 \%$ for HCT-116 and 29.0\% for HT-29 after 24 hours treatment, compared to the single drug treatments and the untreated controls (Figure 5). We also observed a remarkable decrease in the G1 phase and an increase in the population at the G2/M phase after the combination treatment (Figure 5). Both HCT-116 and HT-29 showed the same trend. Compared to BEZ235, PTX seemed to have a greater effect on both cancer cell lines and remarkably increased sub-G1 cell population at both doses (Figure 5).

\section{Combination treatment caused moderate early apoptosis but remarkable decrease in $\mathrm{Bcl}-2$}

We further assayed the effect of PTX and BEZ235 combination treatment on cell death using the Annexin V apoptosis kit. As shown in Figure 6, both PTX and BEZ235 caused some apoptotic cell death and slight early apoptosis after 24 hours treatment. PTX alone at $4 \mathrm{nM}$ resulted in $5.8 \%$ of apoptotic cell death in HCT-116 (2.6\% in the control). Treatment with BEZ235 alone at $50 \mathrm{nM}$ led to $4.8 \%$ apoptotic cell death, whereas the combinatorial application of both agents produced a greater effect, resulting in $9.2 \%$ apoptotic cell death in HCT-116 cells. The trend was less obvious in HT-29 cells (Figure 6B).

From the results obtained earlier, it seems that Annexin V apoptotic cell death was not the major avenue for BEZ235, 

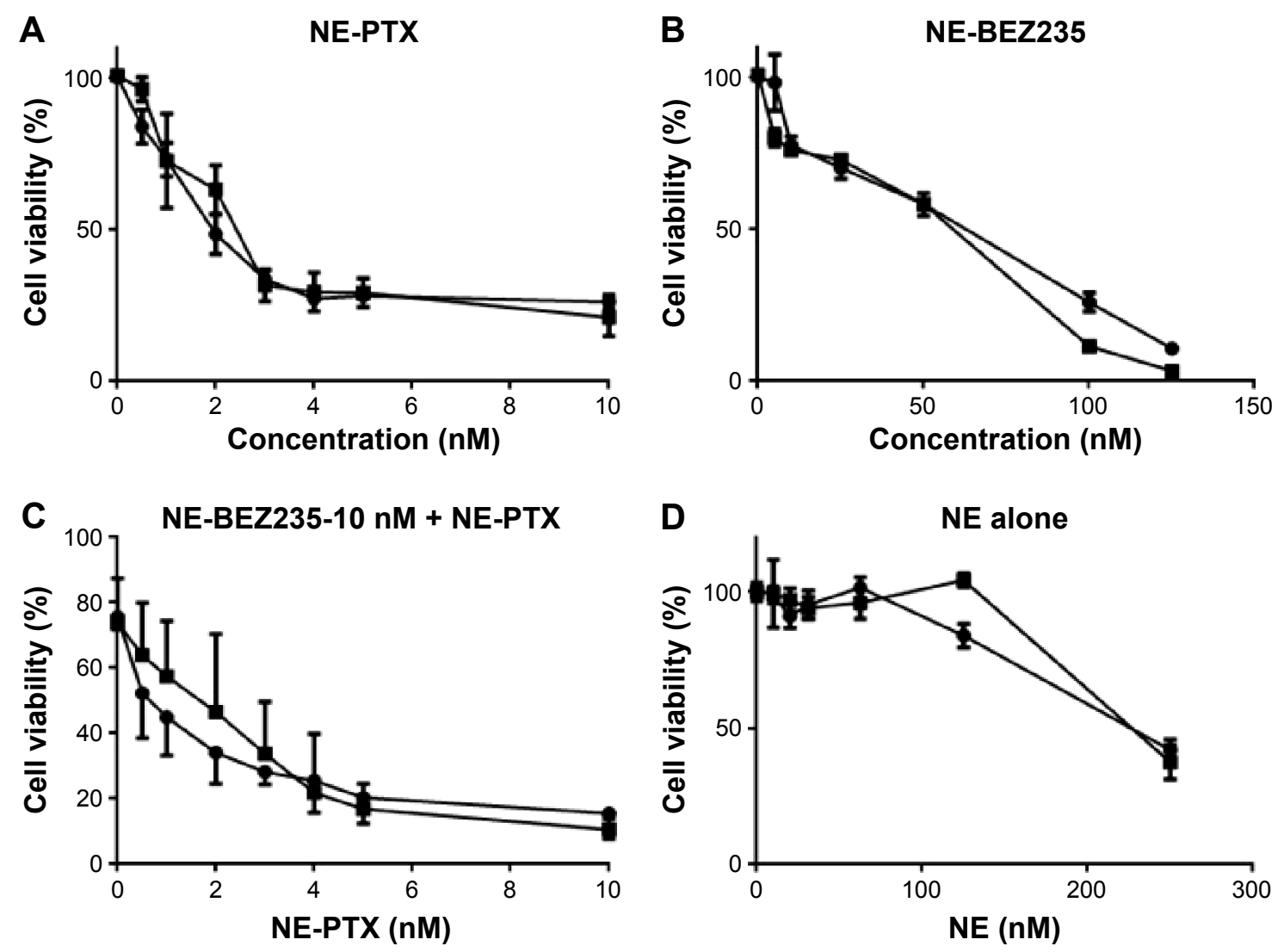

HCT-116

HT-29

Figure 3 Dose-dependent inhibitory effect of NE-loaded PTX and BEZ235 on colon cancer cells.

Notes: The MTT assay results show the effect of NE-PTX and NE-BEZ235 on HCT-II6 and HT-29 cells. (A) Effect of NE-PTX. (B) Effect of NE-BEZ235. (C) Effect of the combination treatment with the two drugs. (D) Effect of unloaded NE nanoparticles.

Abbreviations: MTT, 3-(4,5-dimethylthiazol-2-yl)-2,5-diphenyltetrazolium bromide; NE, nanoemulsion; PTX, paclitaxel.

and PTX caused cell death. We then further analyzed the levels of Bcl-2 protein (an anti-apoptosis protein associated with mitochondria apoptosis pathways) and found that the treatment with a single drug led to a reduction in this protein, but the most significant reduction was seen in the combination treatment (Figure 7), which is consistent with the above cell death results.

\section{Discussion}

PTX is a common chemotherapeutic for several cancers and is also currently being explored as a treatment approach for other cancers including colon cancer. BEZ235 is also a promising chemotherapy drug in clinical trials for many cancers such as colon cancer. Though combination therapies are commonly used against colon cancer, a combination therapy with these two drugs has not been previously reported, especially in the NE format. Here, we show that a combination of these two drugs produces a synergistic inhibition effect on both colon cancer HCT-116 and HT-29 cell lines. Using the free drugs, we showed that with a concentration of $25 \mathrm{nM} \mathrm{BEZ235} \mathrm{(the}$ IC50 is $>120 \mathrm{nM}$ ), PTX could kill 50\% cancer cells at a dose of $3.22 \mathrm{nM}$ for HCT-116 and $3.92 \mathrm{nM}$ for HT-29, which are three times lower than with PTX alone. We further demonstrated that with our NE delivery system, at a concentration as low as $10 \mathrm{nM}$ of BEZ235, PTX could kill 50\% of the colon cancer cells at a dose of $0.69 \mathrm{nM}$ for HCT-116 and $1.7 \mathrm{nM}$ for HT-29. This is a significant improvement in the effectiveness of this combination therapy, which will reduce the side effects of this chemotherapy in colon cancer treatment. Currently, we are investigating the efficacy of this treatment in vivo using a xenotransplant tumor animal model, potentially leading to its application clinically.

The cytotoxicity of PTX has been well studied; it interferes with the process of the breakdown of microtubules during cell division and can cause apoptosis of treated cells. ${ }^{3,4,16}$ The side effects of PTX include hypersensitivity reactions, myelosuppression, and peripheral neuropathy.,6 It can also cause ovarian damage and, thus, interfere with 

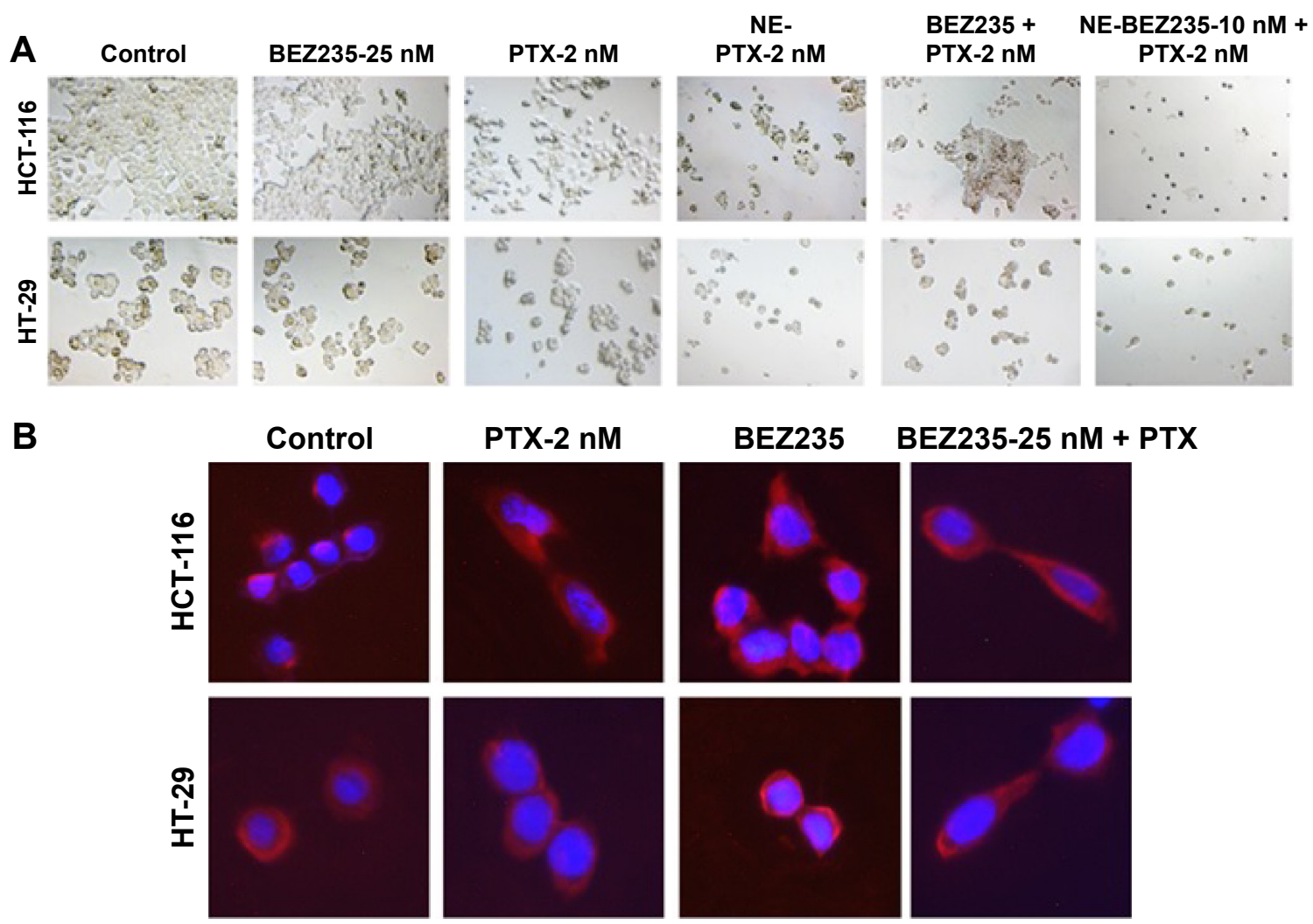

Figure 4 Cell death and morphology changes after BEZ235 and PTX treatment.

Notes: (A) White-field images of the cell death caused by PTX and BEZ235 treatment alone or combination treatment after 24 hours. The dead cells and decreased cell numbers were clear in treated groups compared to the controls. (B) Cell morphology changes after 2 hours treatment with PTX or BEZ235 or both. All images were taken using an Olympus IX5I fluorescent microscope at $100 \times$ magnification.

Abbreviations: NE, nanoemulsion; PTX, paclitaxel.

fertility. ${ }^{36}$ To reduce these side effects but maintain its effectiveness to cancer cells, common approaches include the use of combination treatments with other drugs or the use of effective drug delivery systems. In this study, we used BEZ235 in combination with PTX and used NE to deliver these drugs in order to increase its solubility and decrease the dosage with the same cytotoxicity to cancer cells.

Nanoparticles are extensively used for drug delivery to increase drug efficacy and reduce side effects. In our study, we showed that NE-delivered PTX increased cytotoxicity to HCT-116 and HT-29 markedly. This may be due to the endocytosis effect. In this process, NE-PTX can be easily engulfed by HCT-116 and HT-29 cells as a particle, which contains more drug molecules than free drug as described for other nanoparticles. ${ }^{37}$ This process facilitates PTX entry to cells and induction of higher cytotoxicity. So far, NE-PTX has been tested only with the drugs ceramide ${ }^{14}$ and curcumin ${ }^{11,38}$ as a combination treatment for ovarian and brain cancer cells, in which PTX has been shown to have a synergistic effect with these drugs. In our study, we examined the effect of NE-PTX on HCT-116 and HT-29 colon cancer cells in combination with NE-BEZ235, and we found a similar synergistic effect. All these data suggest that NE-PTX is a good candidate for combinational therapy with a broad range of other drugs.

The rationale behind co-applying PTX and the PI3K/Akt/ mTOR pathway inhibitor BEZ235 is based on the observation that both drugs effectively inhibit colon cancer. ${ }^{19,28}$ It has been shown that PTX can induce a synergistic effect with a few other drugs on a few different cancers. ${ }^{7,31}$ We believed that it would have a similar effect on colon cancer. Chen et $\mathrm{al}^{39,40}$ have shown that the activation of the PI3K/Akt pathway can reduce the effect of chemotherapeutic agents in colon cancer cell lines, and inhibition of this pathway can increase the sensitivity of HT-29 cells to 5-FU. Similarly, leptin has been shown to increase colon cancer resistance to 5-FU treatment via activation of the PI3K/Akt pathway. ${ }^{41}$ Mueller et al studied the effect of BEZ235 and irinotecan on HT-29 cells and showed that BEZ235 increased the effect of irinotecan. ${ }^{42}$ This study indicates that inhibition of the PI3K/Akt/mTOR pathway can be combined with a 
A
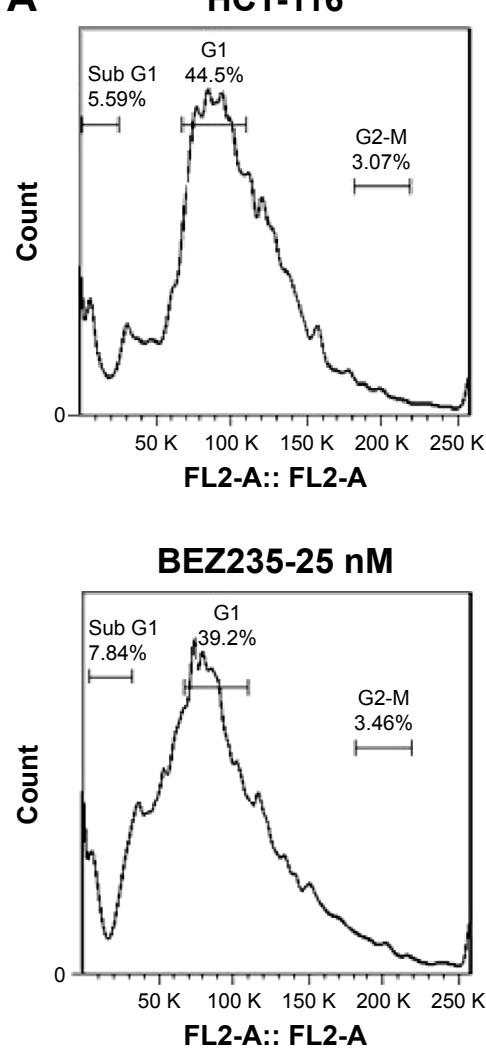

B
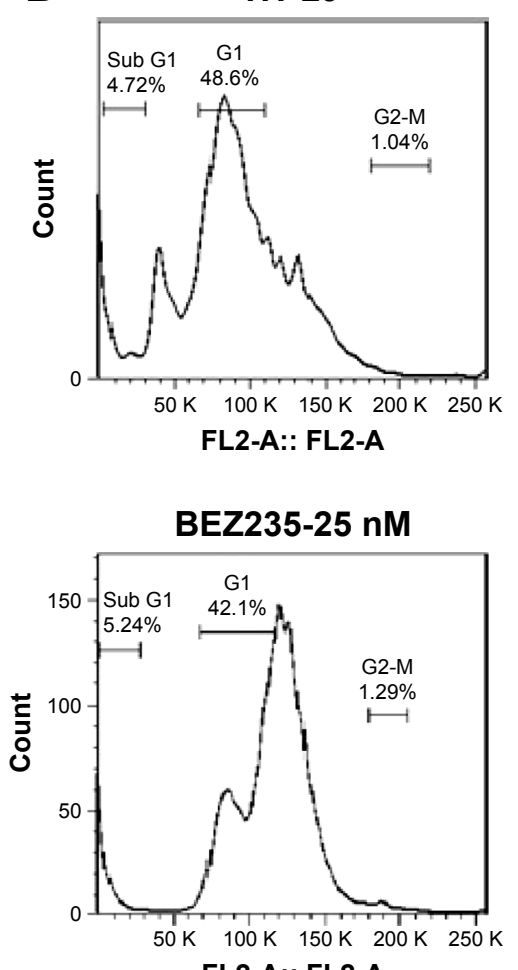

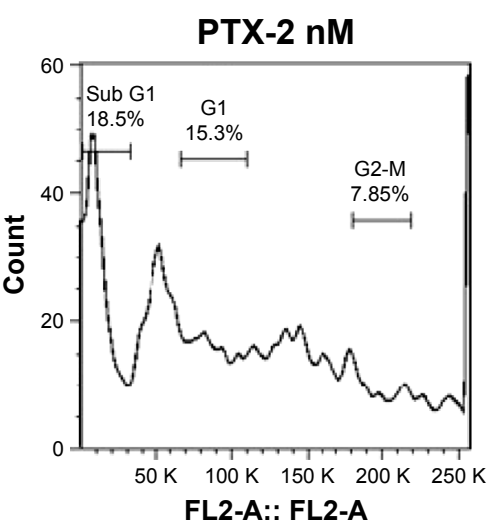

FL2-A:: FL2-A

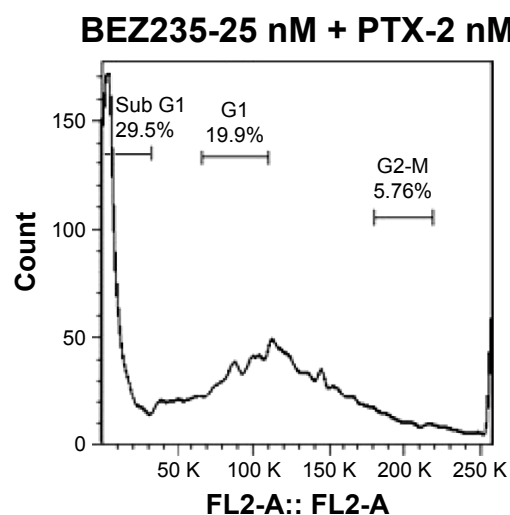

PTX-2 nM

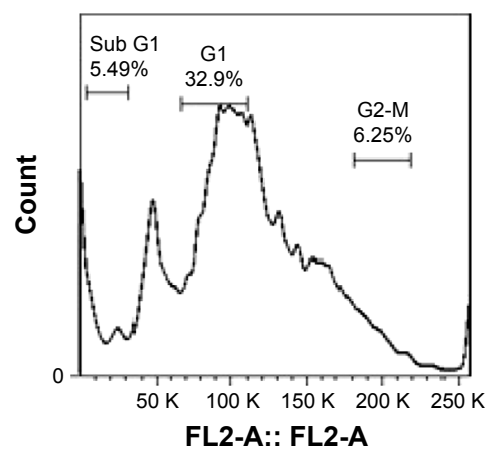

BEZ235-25 nM + PTX-2 nM

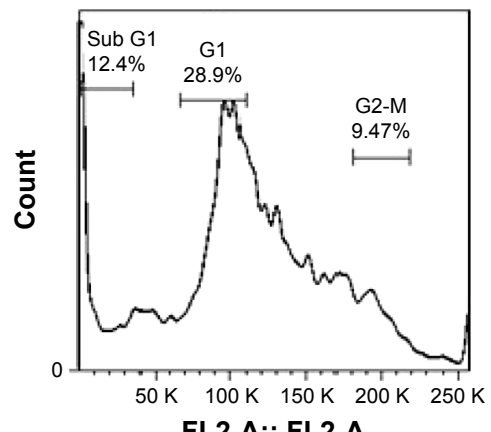

PTX-4 nM

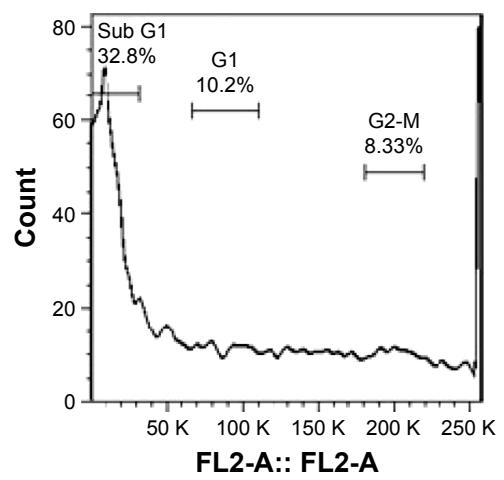

BEZ235-25 nM + PTX-4 nM

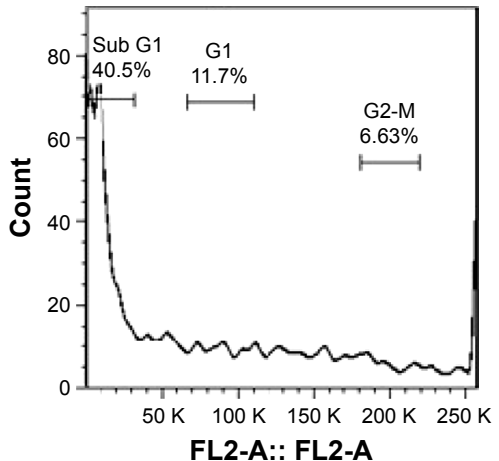

PTX-4 nM

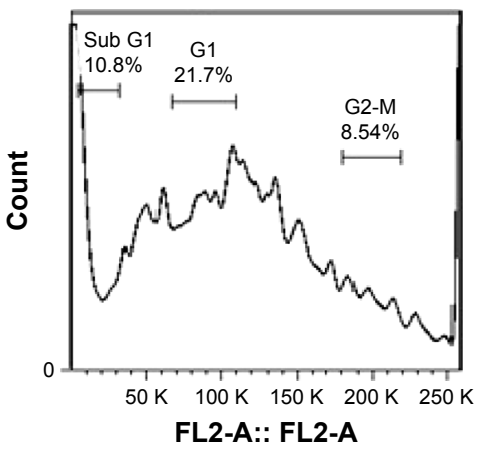

BEZ235-25 nM + PTX-4 nM

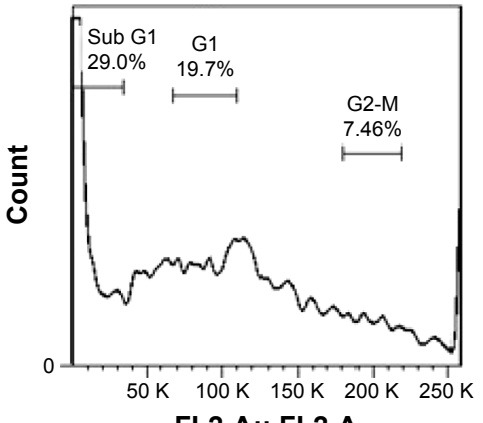

Figure 5 Cell cycle analysis of HCT-II6 and HT-29 cells treated with BEZ235 and PTX.

Notes: The flow cytometry result shows the cell cycle analysis of DNA contents labeled by PI, which is shown by the FL2 channel. Single cells were gated by FSC-H versus FSC-A. All other analyses were based on single populations. (A) Analysis on HCT-1 16 cells. (B) Analysis on HT-29 cells.

Abbreviations: FL2-A, PI channel; FSC-A, forward scatter-area; FSC-H, forward scatter-height; PI, propidium iodide; PTX, paclitaxel. 

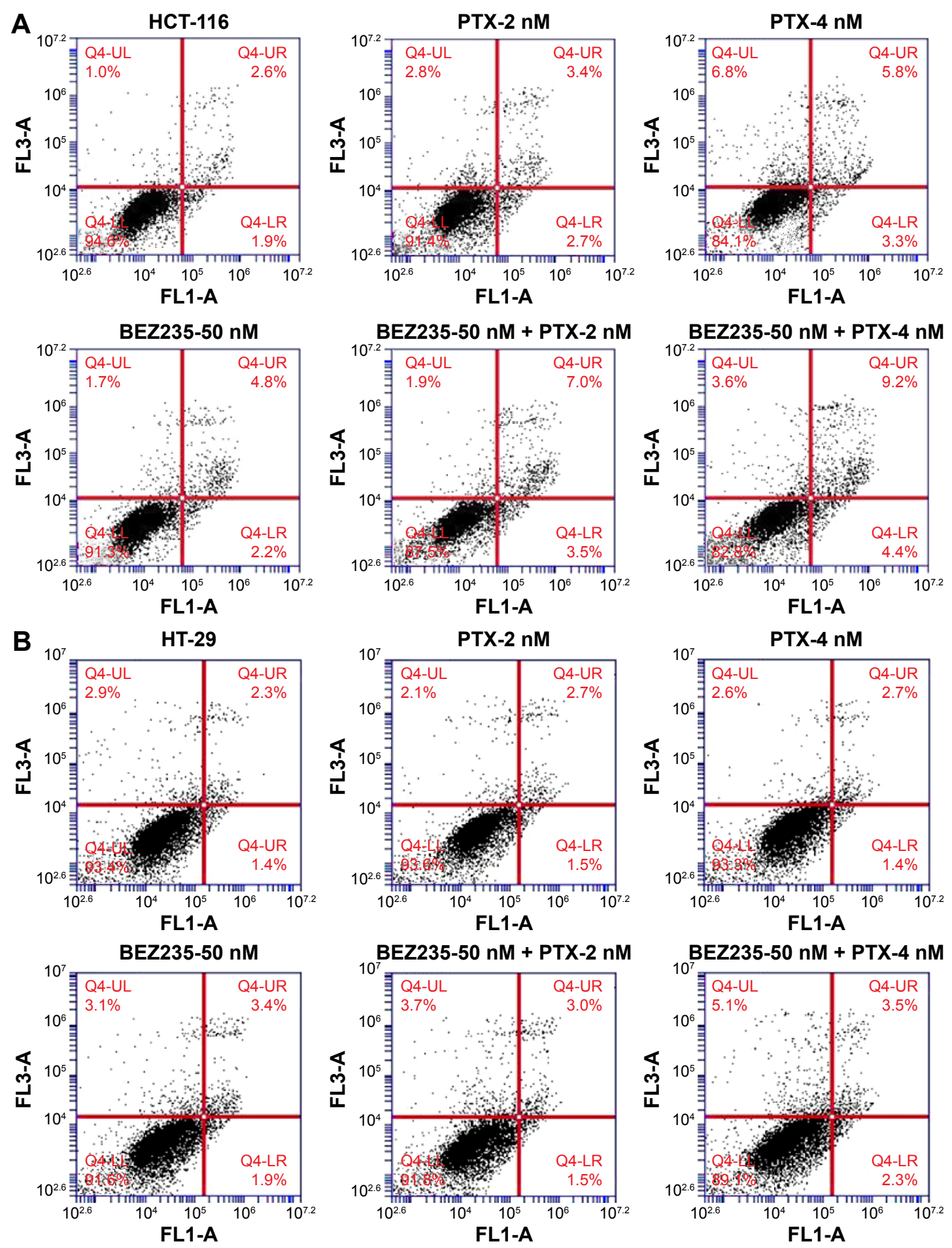

Figure 6 Annexin $\mathrm{V}$ apoptosis analysis for the combination treatment of BEZ235 and PTX.

Notes: The flow cytometry analysis of apoptotic cell death shows that the cotreatment of BEZ235 and PTX has induced more cell death in (A) HCT-I I6 cells and (B) HT-29 cells than any single drug treatment at different doses of $2 \mathrm{nM}$ and $4 \mathrm{nM}$ for PTX and $50 \mathrm{nM}$ for BEZ235.

Abbreviations: FLI-A, Annexin V staining; FL3-A, propidium iodide staining; LL, lower left; LR, lower right; PTX, paclitaxel; Q4, quarter 4; UL, upper left; UR, upper right.

chemotherapeutic agent to increase treatment efficacy in colon cancer. Therefore, we wanted to examine whether BEZ235 treatment could sensitize colon cancer cells to PTX, and our results confirmed that this was true.
Our data indicated that the dual inhibitor of the PI3K/Akt/ mTOR pathway, BEZ235, can sensitize both HCT-116 and HT-29 cells to PTX, especially in the NE-loaded format. This is a new approach for the treatment of colon cancer. HCT-116 


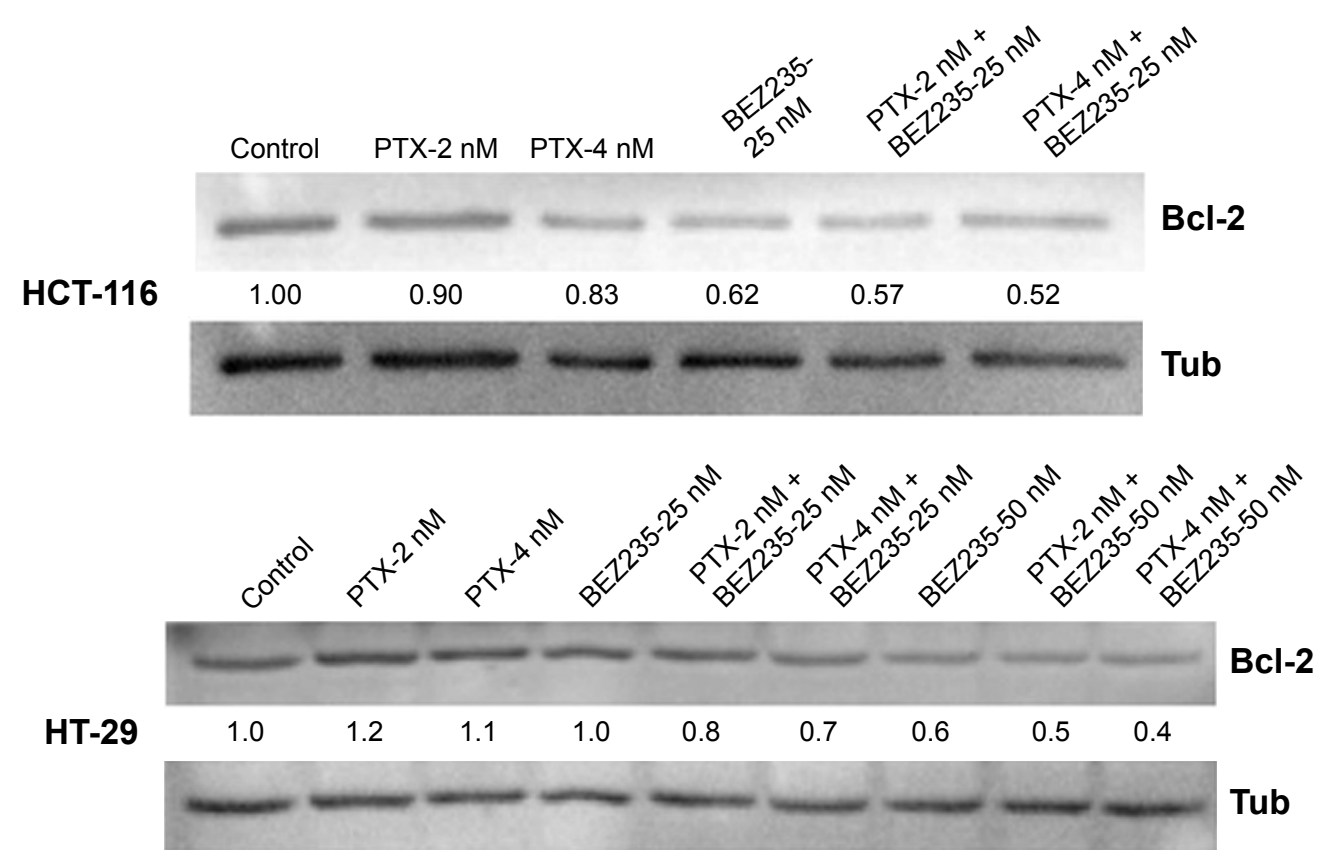

Figure $7 \mathrm{Bcl}-2$ Western-blot analysis for the combination treatment of BEZ235 and PTX.

Note: Combination treatment of BEZ235 and PTX decreased Bcl-2 protein levels detected by Western blot in both HCT-I I6 and HT-29 colon cancer cells. Abbreviations: Bcl-2, B-cell lymphoma 2; Tub, human $\alpha$-tubulin; PTX, paclitaxel.

cells have a mutation in PIK3CA; therefore, their PI3K pathway is highly activated and thus sensitive to the inhibition of the pathway. HT-29 cells, even without PIK3CA mutation, are also sensitive to BEZ235. In addition, HCT-116 cells were reported to have $K$-Ras mutations but not in HT-29 cells. ${ }^{43}$ However, both of them are equally sensitive to the combination treatment, suggesting that the combination therapy with PTX and BEZ235 can be widely applied in colon cancer treatment. BEZ235 has been used for several clinical trials in colon cancer treatment. The approach we tested could be applicable for clinical tests, as both BEZ235 and PTX are approved for clinical use as anticancer agents. BEZ235 is known to have minor side effects, but several studies have shown that it has good tolerance in clinical trials. In this study, we showed that $\mathrm{NE}$ is a low-toxicity nanoparticle delivery system. At the concentration of $200 \mu \mathrm{g} / \mathrm{mL}$, it produced no toxicity to cells. Therefore, this is a promising drug delivery system for future application in combination therapy with BEZ235. A potential future application of this delivery system would be attempting to combine the two drugs in one NE nanoparticle.

Dual inhibition of PI3K/Akt/mTOR using PI-103 has been shown to increase the efficacy of 5-FU in both in vitro and in vivo studies in gastric cancer. ${ }^{44}$ In comparison with single inhibitors of PI3K and mTOR, PI-103 produced an enhanced effect. PI-103 is the first identified dual inhibitor of the PI3K/Akt/mTOR pathway. Although it has a strong antitumor effect, it is not suitable for clinical application due to its high toxicity. ${ }^{45-47}$ In contrast, BEZ235 has been extensively tested in clinical trials for many types of cancers and has demonstrated high effectiveness and low toxicity. ${ }^{26-28,48-50} \mathrm{In}$ the present study, we applied BEZ235 in colon cancer cells HCT-116 and HT-29 in combination with PTX and NE-PTX to demonstrate their combination effect.

Our study has partially elucidated the mechanisms for the combination effect of BEZ235 and PTX. In the cell cycle analysis, the combination treatment produced many more sub-G1 apoptotic cells than with BEZ235 or PTX alone, indicating the synergistic effect on cell death of the two drugs. Moderate cell cycle arrest was observed in the $\mathrm{G} 2 / \mathrm{M}$ phase and a remarkable decrease in the G1 phase. This is different from a previous study, showing that BEZ235 caused G1 arrest in PC3M cells. ${ }^{34}$ The difference could be due to the different cell types and the drug doses used; in the mentioned study, $10 \mathrm{nM}$ and $50 \mathrm{nM} \mathrm{BEZ235}$ were used. Our combination treatment resulted in a reduction in mitochondrial anti-apoptotic protein Bcl-2. Bcl-2 reduction can lead to increased apoptosis. Therefore, both cell proliferation and cell apoptosis are affected by the combination treatment. Further studies are warranted so that the combination technique can be extended to clinical trials.

\section{Acknowledgments}

The authors acknowledge the funding support of the Australia Research Council (ID: DP120100240) to WYG. The authors thank Ms Jennifer Schoning for her kind help in reading and correcting the grammar of this manuscript. 


\section{Disclosure}

The authors report no conflicts of interest in this work.

\section{References}

1. Davies JM, Goldberg RM. Treatment of metastatic colorectal cancer. Semin Oncol. 2011;38(4):552-560.

2. Saville M, Lietzau J, Pluda J, et al. Treatment of HIV-associated Kaposi's sarcoma with paclitaxel. Lancet. 1995;346(8966):26-28.

3. Ahmed AA, Wang X, Lu Z, et al. Modulating microtubule stability enhances the cytotoxic response of cancer cells to paclitaxel. Cancer Res. 2011;71(17):5806-5817.

4. Mielgo A, Torres VA, Clair K, Barbero S, Stupack DG. Paclitaxel promotes a caspase 8-mediated apoptosis through death effector domain association with microtubules. Oncogene. 2009;28(40):3551-3562.

5. Moktan S, Ryppa C, Kratz F, Raucher D. A thermally responsive biopolymer conjugated to an acid-sensitive derivative of paclitaxel stabilizes microtubules, arrests cell cycle, and induces apoptosis. Invest New Drugs. 2012;30(1):236-248.

6. Gupta N, Hatoum H, Dy GK. First line treatment of advanced nonsmall-cell lung cancer - specific focus on albumin bound paclitaxel. Int J Nanomedicine. 2014;9:209-221.

7. Zhang C, Awasthi N, Schwarz MA, Schwarz RE. The dual PI3K/mTOR inhibitor NVP-BEZ235 enhances nab-paclitaxel antitumor response in experimental gastric cancer. Int J Oncol. 2013;43(5):1627-1635.

8. Palumbo R, Sottotetti F, Trifiro G, et al. Nanoparticle albumin-bound paclitaxel (nab-paclitaxel) as second-line chemotherapy in HER2negative, taxane-pretreated metastatic breast cancer patients: prospective evaluation of activity, safety, and quality of life. Drug Des Devel Ther. 2015;9:2189-2199.

9. Peltier S, Oger J-M, Lagarce F, Couet W, Benoît J-P. Enhanced oral paclitaxel bioavailability after administration of paclitaxel-loaded lipid nanocapsules. Pharm Res. 2006;23(6):1243-1250.

10. Bu H, He X, Zhang Z, Yin Q, Yu H, Li Y. A TPGS-incorporating nanoemulsion of paclitaxel circumvents drug resistance in breast cancer. Int J Pharm. 2014;471(1-2):206-213.

11. Ganta S, Amiji M. Coadministration of paclitaxel and curcumin in nanoemulsion formulations to overcome multidrug resistance in tumor cells. Mol Pharm. 2009;6(3):928-939.

12. Choudhury H, Gorain B, Karmakar S, et al. Improvement of cellular uptake, in vitro antitumor activity and sustained release profile with increased bioavailability from a nanoemulsion platform. Int J Pharm. 2014;460(1-2):131-143.

13. Dias ML, Carvalho JP, Rodrigues DG, Graziani SR, Maranhao RC. Pharmacokinetics and tumor uptake of a derivatized form of paclitaxel associated to a cholesterol-rich nanoemulsion (LDE) in patients with gynecologic cancers. Cancer Chemother Pharmacol. 2007;59(1): 105-111.

14. Desai A, Vyas T, Amiji M. Cytotoxicity and apoptosis enhancement in brain tumor cells upon coadministration of paclitaxel and ceramide in nanoemulsion formulations. J Pharm Sci. 2008;97(7):2745-2756.

15. Deepa G, Ashwanikumar N, Pillai JJ, Kumar GS. Polymer nanoparticles - a novel strategy for administration of paclitaxel in cancer chemotherapy. Curr Med Chem. 2012;19(36):6207-6213.

16. Guo XY, Wang P, Du QG, et al. Paclitaxel and gemcitabine combinational drug-loaded mucoadhesive delivery system in the treatment of colon cancers. Drug Res. 2015;65(4):199-204.

17. Goncalves A, Braguer D, Carles G, Andre N, Prevot C, Briand C. Caspase-8 activation independent of CD95/CD95-L interaction during paclitaxel-induced apoptosis in human colon cancer cells (HT29-D4). Biochem Pharmacol. 2000;60(11):1579-1584.

18. Feng SS, Mu L, Win KY, Huang G. Nanoparticles of biodegradable polymers for clinical administration of paclitaxel. Curr Med Chem. 2004; 11(4):413-424.

19. Xu R, Sato N, Yanai K, et al. Enhancement of paclitaxel-induced apoptosis by inhibition of mitogen-activated protein kinase pathway in colon cancer cells. Anticancer Res. 2009;29(1):261-270.
20. Chen J. Potential value and limitation of dual inhibitors of PI3K and mTOR in the treatment of cancer. Curr Cancer Drug Targets. 2013;13(2): $117-120$.

21. Bu Z, Ji J. Therapeutic implications of mTOR inhibitors in the treatment of gastric cancer. Curr Cancer Drug Targets. 2013;13(2):121-125.

22. Cho DC, Mier JW. Dual inhibition of PI3-kinase and mTOR in renal cell carcinoma. Curr Cancer Drug Targets. 2013;13(2):126-142.

23. Elfiky AA, Jiang $Z$. The PI 3 kinase signaling pathway in prostate cancer. Curr Cancer Drug Targets. 2013;13(2):157-164.

24. Page C, Lin HJ, Jin Y, et al. Overexpression of Akt/AKT can modulate chemotherapy-induced apoptosis. Anticancer Res. 2000;20(1): 407-416.

25. Brognard J, Clark AS, Ni Y, Dennis PA. Akt/protein kinase B is constitutively active in non-small cell lung cancer cells and promotes cellular survival and resistance to chemotherapy and radiation. Cancer Res. 2001;61(10):3986-3997.

26. Chapuis N, Tamburini J, Green AS, et al. Dual inhibition of PI3K and mTORC1/2 signaling by NVP-BEZ235 as a new therapeutic strategy for acute myeloid leukemia. Clin Cancer Res. 2010;16(22): $5424-5435$.

27. Manara MC, Nicoletti G, Zambelli D, et al. NVP-BEZ235 as a new therapeutic option for sarcomas. Clin Cancer Res. 2010;16(2):530-540.

28. Wu P, Hu Y-Z. PI3K/Akt/mTOR pathway inhibitors in cancer: a perspective on clinical progress. Curr Med Chem. 2010;17(35):4326-4341.

29. Roper J, Richardson MP, Wang WV, et al. The dual PI3K/mTOR inhibitor NVP-BEZ235 induces tumor regression in a genetically engineered mouse model of PIK3CA wild-type colorectal cancer. PLOS One. 2011;6(9): 25132.

30. Chen J, Shao R, Li L, Xu ZP, Gu W. Effective inhibition of colon cancer cell growth with MgAl-layered double hydroxide (LDH) loaded 5-FU and PI3K/mTOR dual inhibitor BEZ-235 through apoptotic pathways. Int J Nanomedicine. 2014;9:3403-3411.

31. Kudoh A, Oishi T, Itamochi H, et al. Dual inhibition of phosphatidylinositol 3'-kinase and mammalian target of rapamycin using NVPBEZ235 as a novel therapeutic approach for mucinous adenocarcinoma of the ovary. Int J Gynecol Cancer. 2014;24(3):444-453.

32. Skoura E, Syrigos KN, Saif MW. Preclinical research in treatment of pancreatic cancer. JOP. 2013;14(4):384-387.

33. Lin SF, Huang YY, Lin JD, Chou TC, Hsueh C, Wong RJ. Utility of a PI3K/mTOR inhibitor (NVP-BEZ235) for thyroid cancer therapy. PLoS One. 2012;7(10):e46726.

34. Maira S-M, Stauffer F, Brueggen J, et al. Identification and characterization of NVP-BEZ235, a new orally available dual phosphatidylinositol 3-kinase/mammalian target of rapamycin inhibitor with potent in vivo antitumor activity. Mol Cancer Ther. 2008;7(7):1851-1863.

35. Wang TH, Chan YH, Chen CW, et al. Paclitaxel (Taxol) upregulates expression of functional interleukin-6 in human ovarian cancer cells through multiple signaling pathways. Oncogene. 2006;25(35): 4857-4866.

36. Ozcelik B, Turkyilmaz C, Ozgun MT, et al. Prevention of paclitaxel and cisplatin induced ovarian damage in rats by a gonadotropinreleasing hormone agonist. Fertil Steril. 2010;93(5):1609-1614.

37. Choi SJ, Choy JH. Layered double hydroxide nanoparticles as targetspecific delivery carriers: uptake mechanism and toxicity. Nanomedicine (Lond). 2011;6(5):803-814.

38. Ganta S, Devalapally H, Amiji M. Curcumin enhances oral bioavailability and anti-tumor therapeutic efficacy of paclitaxel upon administration in nanoemulsion formulation. J Pharm Sci. 2010;99(11): 4630-4641.

39. Chen JHX, Qiao L, Katsifis A. Insulin caused drug resistance to oxaliplatin in colon cancer cell HT29. J Gastrointest Oncol. 2011;2:27-33.

40. Chen J, Katsifis A, Hu C, Huang XF. Insulin decreases therapeutic efficacy in colon cancer cell line HT29 via the activation of the PI3K/ Akt pathway. Curr Drug Discov Technol. 2011;8(2):119-125.

41. Bartucci M, Svensson S, Ricci-Vitiani L, et al. Obesity hormone leptin induces growth and interferes with the cytotoxic effects of 5-fluorouracil in colorectal tumor stem cells. Endocr Relat Cancer. 2010;17(3): 823-833. 
42. Mueller A, Bachmann E, Linnig M, et al. Selective PI3K inhibition by BKM120 and BEZ235 alone or in combination with chemotherapy in wild-type and mutated human gastrointestinal cancer cell lines. Cancer Chemother Pharmacol. 2012;69(6):1601-1615.

43. Rajesh D, Schell K, Verma A. Ras mutation, irrespective of cell type and p53 status, determines a cell's destiny to undergo apoptosis by okadaic acid, an inhibitor of protein phosphatase 1 and 2A. Mol Pharmacol. 1999;56(3):515-525.

44. Bhattacharya B, Akram M, Balasubramanian I, et al. Pharmacologic synergy between dual phosphoinositide-3-kinase and mammalian target of rapamycin inhibition and 5-fluorouracil in PIK3CA mutant gastric cancer cells. Cancer Biol Ther. 2012;13(1):34-42.

45. Zou Z-Q, Zhang X-H, Wang F, et al. A novel dual PI3K $\alpha / \mathrm{mTOR}$ inhibitor PI-103 with high antitumor activity in non-small cell lung cancer cells. Int J Mol Med. 2009;24(1):97-101.

46. Park S, Chapuis N, Bardet V, et al. PI-103, a dual inhibitor of Class IA phosphatidylinositide 3-kinase and mTOR, has antileukemic activity in AML. Leukemia. 2008;22(9):1698-1706.
47. Schwab J, Antonescu C, Boland P, et al. Combination of PI3K/mTOR inhibition demonstrates efficacy in human chordoma. Anticancer Res. 2009;29(6):1867-1871.

48. Santiskulvong C, Konecny GE, Fekete M, et al. Dual targeting of phosphoinositide 3-kinase and mammalian target of rapamycin using NVP-BEZ235 as a novel therapeutic approach in human ovarian carcinoma. Clin Cancer Res. 2011;17(8):2373-2384.

49. Wander SA, Hennessy BT, Slingerland JM. Next-generation mTOR inhibitors in clinical oncology: how pathway complexity informs therapeutic strategy. J Clin Invest. 2011;121(4):1231-2141.

50. Serra V, Scaltriti M, Prudkin L, et al. PI3K inhibition results in enhanced HER signaling and acquired ERK dependency in HER2-overexpressing breast cancer. Oncogene. 2011;30(22):2547-2557.
International Journal of Nanomedicine

\section{Publish your work in this journal}

The International Journal of Nanomedicine is an international, peerreviewed journal focusing on the application of nanotechnology in diagnostics, therapeutics, and drug delivery systems throughout the biomedical field. This journal is indexed on PubMed Central, MedLine, CAS, SciSearch ${ }^{\circledR}$, Current Contents ${ }^{\circledR} /$ Clinical Medicine,

\section{Dovepress}

Journal Citation Reports/Science Edition, EMBase, Scopus and the Elsevier Bibliographic databases. The manuscript management system is completely online and includes a very quick and fair peer-review system, which is all easy to use. Visit http://www.dovepress.com/ testimonials.php to read real quotes from published authors. 